\title{
Simplified Protocol of Quantum Teleportation
}

\author{
Mario Mastriani \\ Quantum Optics Department, Merx Lab, 2875 NE 191, Aventura, FL 33180, USA. \\ Applied Physics Group, Merx-FI, 650 NE 64th - GPH7, Miami, FL 33138, USA.
}

ORCID Id: 0000-0002-5627-3935

mmastri@merxcomm.com

A simplified version of the quantum teleportation protocol is presented in here. Its experimental confirmation will have deep implications for a better understanding of Quantum Entanglement with a particular projection on Quantum Communications.

Keywords-Bell states; BSM; EPR pairs; LOCC; No-Cloning Theorem; Quantum Communications; Quantum Entanglement; Quantum Teleportation.

PACS numbers: 03.67.Hk, 03.65.Ud, 03.67.-a, 03.67.Lx, 03.65.Yz

\section{Introduction}

First, we should mention the key pieces in Quantum Information Processing (in general), and Quantum Computing, and Communications (in particular), i.e., the Principle of Superposition and the quantum entanglement [1]. Both closely related to the work of Erwin Schrödinger [2] [3]. In fact, Schrödinger defined the entangled of pure states as the pure quantum state $\left|\Psi_{A B}\right\rangle$ associated to composite systems like $A$ and $B$ that cannot be represented in the form of simple tensor products of subsystem state-vectors, that is [1],

$$
\left|\Psi_{A B}\right\rangle \neq\left|\psi_{A}\right\rangle \otimes\left|\psi_{B}\right\rangle
$$

where “ $\otimes$ " indicates the tensor product (also known as Kronecker's product) while $\left|\psi_{A}\right\rangle$ and $\left|\psi_{B}\right\rangle$ are vectors providing the states of both subsystems, such as elementary particles [2] [3]. Those states of composite systems that can be represented as tensor products of subsystem states constitute the complement in the set of pure states, the product states [1]. In fact, states of the composite system that can be represented in this form are called separable states. Then, since not all states are separable states (and thus product states) we will carry out the following analysis. Establish a pair of basis: $\left\{\left|x_{A}\right\rangle\right\}$ for $H_{\mathrm{A}}$ and $\left\{\left|y_{B}\right\rangle\right\}$ for $H_{\mathrm{B}}$. In $H_{\mathrm{A}} \otimes H_{\mathrm{B}}$, the most general state is of the form

$\left|\Psi_{A B}\right\rangle=\sum_{x, y} n_{x y}\left|x_{A}\right\rangle \otimes\left|y_{B}\right\rangle$.

Therefore, this state will be separable if there exist vectors $\left[n_{x}^{A}\right],\left[n_{y}^{B}\right]$ so that $n_{x y}=n_{x}^{A} n_{y}^{B}$ yielding $\left|\psi_{A}\right\rangle=\sum_{x} n_{x}^{A} x_{A}$ and $\left|\psi_{B}\right\rangle=\sum_{y} n_{y}^{B} y_{B}$. It is inseparable if for any vectors $\left[n_{x}^{A}\right],\left[n_{y}^{B}\right]$ at least for one pair of coordinates $n_{x}^{A}, n_{y}^{B}$ we have $n_{x y} \neq n_{x}^{A} n_{y}^{B}$. If a state is inseparable, it is called an entangled state.

On the other hand, in 1935 Albert Einstein, Boris Podolsky and Nathan Rosen (EPR) propose a thought experiment by which they try to demonstrate that the wave-function does not provide a complete description of physical reality (and which gives rise to the famous EPR paradox), and hence 
that the Copenhagen interpretation is unsatisfactory; resolutions of the paradox have important implications for the interpretation of quantum mechanics [4]. The essence of the paradox is that particles can interact in such a way that it is possible to measure both their position and their momentum more accurately than Heisenberg's uncertainty principle allows [5], unless measuring one particle instantaneously affects the other to prevent this accuracy, which would involve information being transmitted faster than light [6]-[8] as forbidden by the theory of relativity ("spooky action at a distance") [5] [9]-[13]. This consequence had not previously been noticed and seemed unreasonable at the time; the phenomenon involved is now known as quantum entanglement [1] [5].

Moreover, in 1964 John S. Bell introduces his famous theorem [9] associated with 4 states, i.e., 2qubit vectors into a combined space of Hilbert $\mathrm{H}_{A B}=\mathrm{H}_{A}^{2} \otimes \mathrm{H}_{B}^{2}$, and relative to two subsystems A and $\mathrm{B}$,

$\left|\Phi_{A B}^{ \pm}\right\rangle=\frac{1}{\sqrt{2}}\left(\left|0_{A}, 0_{B}\right\rangle \pm\left|1_{A}, 1_{B}\right\rangle\right), \quad\left|\Psi_{A B}^{ \pm}\right\rangle=\frac{1}{\sqrt{2}}\left(\left|0_{A}, 1_{B}\right\rangle \pm\left|1_{A}, 0_{B}\right\rangle\right)$

where they are called Bell's states, and also known as EPR pairs. This theorem raises an inequality, which when violated by quantum mechanics establishes the non-locality present in the entanglement of two subsystems like A and B. Besides, a posterior redefinition of this inequality due to Clauser, Horne, Shimony and Holt (CHSH) [14] leads to a more conducive way to experimental testing with [13] and without [15] loopholes.

An extremely important concept in quantum teleportation is the No-Cloning Theorem [5] [16], which states that it is impossible to create an identical copy of an arbitrary unknown quantum state. This No-Go Theorem of quantum mechanics was articulated by Wootters and Zurek [16] and Dieks [12] in 1982, and has profound implications in quantum computing and related fields. Besides, the NoCloning Theorem [5] prevents the transfer of information faster than the speed of light [6-8]. We have seen that a measurement on subsystem A instantaneously transforms subsystem B into a well-defined state. The word "instantaneous" is seductive. Let us imagine that an entangled pure state has been produced whose subsystem $S_{A}$ is at Alice's location and whose another subsystem $S_{B}$ at Bob's location, very far away. Alice attempts to transmit one bit of information to Bob by measuring one of two non-commuting observables on her subsystem $\mathrm{S}_{\mathrm{A}}$. If Bob succeeds in reading out this information on his subsystem $S_{B}$, then it would have been transmitted at a velocity greater than that of light and this would contradict the theory of relativity [6].

Quantum teleportation [17]-[19] is a process by which a quantum state $|\psi\rangle$ can be transferred from one point to another while destroying the original state, which is required by the no-cloning theorem [5] [12] [16]. This process takes place between two different locations. An EPR pair (i.e., two maximally entangled states) is apportioned between Alice and Bob. Alice has the state to be teleported. This state is entangled with his EPR state, and after that a process involving quantum gates, Alice performs measurements with a certain probability, so that in identifying the original state in the appropriate base she transmits the elements of that base but in its classic version (i.e., two bits) through a classic channel. On the other side, Bob receives both bits, so that with them and the appropriate quantum gates, he rebuilds the original state. Clearly, we can see that a quantum teleportation depends on a classical communication, which can proceed no faster than the speed of light, it cannot be used for faster-than-light transport or communication of classical bits. Of course, the classical channel is the weak link in this chain in terms of transmission speed.

Otherwise, and since the quantum entanglement has a serious enemy, and in addition, quantum teleportation necessarily depends on the quantum entanglement, then such an enemy is inherited by quantum teleportation. This enemy is decoherence [20], which usually collapse the entanglement. Decoherence can be viewed as the loss of information from a system into the environment, since every system is loosely coupled with the energetic state of its surroundings. This interaction between the state and its environment is clearly seen during the quantum measurement [21], which causes the collapse of the wave-function [20] [21]. To try to mitigate this problem, we must apply reconstructive techniques of the state, which results in an increase in the computational cost of the process as a whole [22]. 
In this paper we present a new protocol of quantum teleportation which does not require any disambiguation based on the transmission of classical bits, as in fact it happens in the original version [12] [17]-[19], which when using two channels (one classic for the bits and another quantum for the distribution of the EPRs) is more vulnerable to attacks. However, the new version is simpler to implement in the laboratory than the original. This possibility has its origin in a work of Quantum Communications [23] which, through an extensive analysis, reaches the conclusion that such a possibility is viable.

Finally, the main pending aspect within the teleportation is its lack of instantaneity (seen and a whole) and a clear definition of its bandwidth [23]. Both are the responsibility of the classic channel that the protocol necessarily uses. To this scenario points to this work based on [23].

\section{Setup}

For the implementation of quantum teleportation, we must use some quantum gates, which we are going to present next. The first one is the Hadamard gate, which acts on a single qubit. It maps the basis state $|0\rangle$ to $1 / \sqrt{2}(|0\rangle+|1\rangle)$ and $|1\rangle$ to $1 / \sqrt{2}(|0\rangle-|1\rangle)$, which means that a measurement will have equal probabilities to become 1 or 0 (i.e., it creates a superposition). It is represented by the Hadamard matrix:

$$
H=\frac{1}{\sqrt{2}}\left(\sigma_{x}+\sigma_{z}\right)=\frac{1}{\sqrt{2}}\left[\begin{array}{cc}
1 & 1 \\
1 & -1
\end{array}\right]
$$

where $\sigma_{x}$ and $\sigma_{z}$ are two of the three Pauli's matrices $\left(\sigma_{x}, \sigma_{y}, \sigma_{z}\right)[24]$

$$
\sigma_{x}=\left[\begin{array}{ll}
0 & 1 \\
1 & 0
\end{array}\right], \quad \sigma_{y}=\left[\begin{array}{cc}
0 & -i \\
i & 0
\end{array}\right] \quad \text { and } \quad \sigma_{z}=\left[\begin{array}{cc}
1 & 0 \\
0 & -1
\end{array}\right]
$$

with $i=\sqrt{-1}$. Figure 1 represents the Hadamard's gate [24].

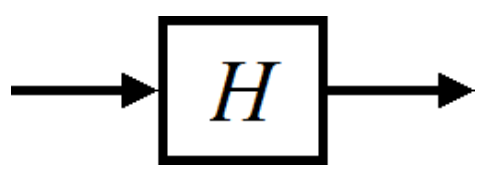

Fig. 1 Hadamard's $(\mathrm{H})$ gate.

The second one is the Controlled NOT gate or simply CNOT, which operates on a quantum register consisting of 2 qubits. The CNOT gate flips the second qubit (the target qubit) if and only if the first qubit (the control qubit) is $|1\rangle$. The inputs are allowed to be a linear superposition of $\{|0\rangle,|1\rangle\}$. As an example of this, we can see that the CNOT gate transforms a superposed quantum state of the kind $a|00\rangle+b|01\rangle+c|10\rangle+d|11\rangle$ into another like $a|00\rangle+b|01\rangle+c|11\rangle+d|10\rangle$. The CNOT gate can be represented by the matrix:

$$
C N O T=|0\rangle\langle 0|\otimes I+| 0\rangle\langle 0| \otimes \sigma_{x}=\left[\begin{array}{cccc}
1 & 0 & 0 & 0 \\
0 & 1 & 0 & 0 \\
0 & 0 & 0 & 1 \\
0 & 0 & 1 & 0
\end{array}\right]
$$

where $I$ is the identity matrix. Figure 2 represents the CNOT gate [24]. 


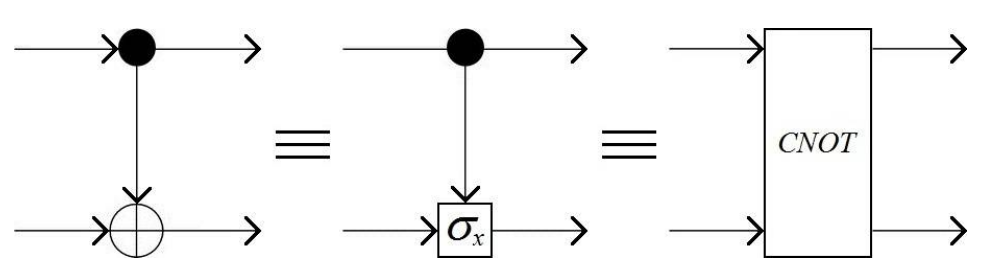

Fig. 2 CNOT gate.

With both gates, we can build the famous Bell state (or EPR pair, named after Einstein, Podolsky, and Rosen paper [4]) from computational basis state $|0\rangle$ [5], as we can see in Figure 3,

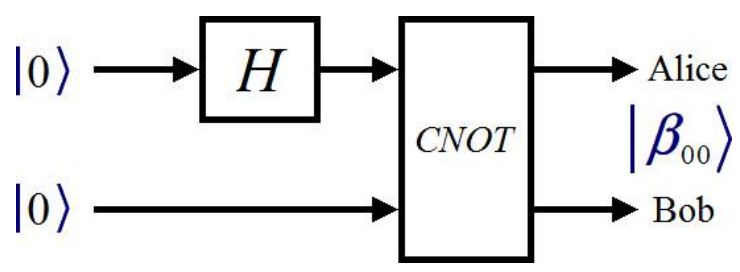

Fig. 3 The output of these gates (Hadamard and CNOT) is an EPR qubit or Bell state. In fact, an EPR pair, one to Alice and other to Bob.

As an example, we are going to build $\left|\beta_{00}\right\rangle$, i.e., the Bell state of Figure 3 based on two states $|0\rangle$ at its entrance and the application of Hadamard $(\mathrm{H})$ and CNOT gates in that order. Thus, for the upper branch of Figure 3, we have

$$
H|0\rangle=\frac{1}{\sqrt{2}}\left[\begin{array}{cc}
1 & 1 \\
1 & -1
\end{array}\right]\left[\begin{array}{l}
1 \\
0
\end{array}\right]=\left[\begin{array}{l}
1 / \sqrt{2} \\
1 / \sqrt{2}
\end{array}\right]
$$

While by the lower branch, simply $|0\rangle=|0\rangle$. Now, and before of CNOT gate, the Kronecker's product “ $\otimes$ " intervenes between both branchs of Figure 3, the upper and the lower,

$$
|0\rangle \otimes H|0\rangle=\left[\begin{array}{l}
1 \\
0
\end{array}\right] \otimes\left[\begin{array}{l}
1 / \sqrt{2} \\
1 / \sqrt{2}
\end{array}\right]=\left[\begin{array}{c}
1 / \sqrt{2} \\
0 \\
1 / \sqrt{2} \\
0
\end{array}\right]
$$

Finally, CNOT gate is applied

$$
\begin{aligned}
{\left[\begin{array}{llll}
1 & 0 & 0 & 0 \\
0 & 1 & 0 & 0 \\
0 & 0 & 0 & 1 \\
0 & 0 & 1 & 0
\end{array}\right]\left[\begin{array}{c}
1 / \sqrt{2} \\
0 \\
1 / \sqrt{2} \\
0
\end{array}\right] } & =\left[\begin{array}{c}
1 / \sqrt{2} \\
0 \\
0 \\
1 / \sqrt{2}
\end{array}\right]=\frac{1}{\sqrt{2}}\left[\begin{array}{l}
1 \\
0 \\
0 \\
0
\end{array}\right]+\frac{1}{\sqrt{2}}\left[\begin{array}{l}
0 \\
0 \\
0 \\
1
\end{array}\right] \\
& =\frac{1}{\sqrt{2}}|0\rangle \otimes|0\rangle+\frac{1}{\sqrt{2}}|1\rangle \otimes|1\rangle=\frac{1}{\sqrt{2}}|0\rangle|0\rangle+\frac{1}{\sqrt{2}}|1\rangle|1\rangle \\
& =\frac{1}{\sqrt{2}}(|00\rangle+|11\rangle)=\left|\beta_{00}\right\rangle
\end{aligned}
$$


The complete set of Bell's basis are:

$$
\begin{aligned}
& \left|\beta_{00}\right\rangle=\left|\Phi^{+}\right\rangle=\frac{1}{\sqrt{2}}(|00\rangle+|11\rangle) \\
& \left|\beta_{01}\right\rangle=\left|\Phi^{-}\right\rangle=\frac{1}{\sqrt{2}}(|00\rangle-|11\rangle) \\
& \left|\beta_{10}\right\rangle=\left|\Psi^{+}\right\rangle=\frac{1}{\sqrt{2}}(|01\rangle+|10\rangle) \\
& \left|\beta_{11}\right\rangle=\left|\Psi^{-}\right\rangle=\frac{1}{\sqrt{2}}(|01\rangle-|10\rangle)
\end{aligned}
$$

then,

$$
\begin{aligned}
& |00\rangle=\frac{1}{\sqrt{2}}\left(\left|\Phi^{+}\right\rangle+\left|\Phi^{-}\right\rangle\right)=\frac{1}{\sqrt{2}}\left(\left|\beta_{00}\right\rangle+\left|\beta_{01}\right\rangle\right) \\
& |01\rangle=\frac{1}{\sqrt{2}}\left(\left|\Psi^{+}\right\rangle+\left|\Psi^{-}\right\rangle\right)=\frac{1}{\sqrt{2}}\left(\left|\beta_{10}\right\rangle+\left|\beta_{11}\right\rangle\right) \\
& |10\rangle=\frac{1}{\sqrt{2}}\left(\left|\Psi^{+}\right\rangle-\left|\Psi^{-}\right\rangle\right)=\frac{1}{\sqrt{2}}\left(\left|\beta_{10}\right\rangle-\left|\beta_{11}\right\rangle\right) \\
& |11\rangle=\frac{1}{\sqrt{2}}\left(\left|\Phi^{+}\right\rangle-\left|\Phi^{-}\right\rangle\right)=\frac{1}{\sqrt{2}}\left(\left|\beta_{00}\right\rangle-\left|\beta_{01}\right\rangle\right)
\end{aligned}
$$

On the other hand and as a complement to the tools seen so far in this section, it is necessary to be able to evaluate the quality of the experimental implementation of teleportation protocols in the presence of noise, i.e., how similar is the teleported state in comparison to the original? Therefore, we will need a good metric to be able to evaluate said transfer quality. Then, we recommend here two versions of the most used metric in teleportation, fidelity [25]. These versions have to do with the characteristic of the state to teleport, i.e., if it is pure or a mixed state [25]-[27].

Fidelity $f$ is a metric that shows us how similar are the initial and final states in a quantum process [25]. It has widely been used to characterize the performance of various quantum information tasks. Fidelity $f$ is bounded by $0 \leq f \leq 1$, where the unit fidelity $(f=1)$ implies that the initial and final states are equivalent [25]. Quantum teleportation is designed to transmit all possible (unknown) input states, thus a measure of averaging over all inputs is used. That is the average fidelity $F$. It quantifies how well the unknown input states can be transmitted to another location. Thus, $F$ shows the optimality of the quantum teleportation. There it is shown that the unit average fidelity $(F=1)$ can be obtained when the two remote parties share the maximally entangled states, whereas $F=2 / 3$ is the maximum fidelity attainable in any classical schemes which cannot use the entanglement [25].

Therefore, if $\left|\psi_{\text {in }}\right\rangle$ and $\left|\psi_{\text {out }}\right\rangle$ are the state to be teleported and the state teleported, respectively,

$$
\rho_{\text {in }}=\left|\psi_{\text {in }}\right\rangle\left\langle\psi_{\text {in }}\right| \text { and } \rho_{\text {out }}=\left|\psi_{\text {out }}\right\rangle\left\langle\psi_{\text {out }}\right|
$$

will be their respective density matrices. Fidelity quantifies a transformation performance between $\left|\psi_{\text {in }}\right\rangle$ and $\left|\psi_{\text {out }}\right\rangle$ states as,

$$
f=\operatorname{Tr}\left[\left(\sqrt{\rho_{\text {out }}} \rho_{\text {in }} \sqrt{\rho_{\text {out }}}\right)^{1 / 2}\right] .
$$

The quantum teleportation applies to unknown input states so that the average fidelity - an average of the fidelities $f$ over all possible input states-is used:

$$
F=\int f d \psi_{\text {in }}
$$


where $d \psi_{\text {in }}$ is Haar measure with $\int d \psi_{\text {in }}=1$. Here, $F=1$ implies that the task is perfectly performed for all possible inputs, while $F=1 / 2$ does at random [25].

\section{Standard Quantum Teleportation}

Then, we will develop the standard quantum teleportation protocol with and without noise. Where, the noiseless analysis is essential when keeping in mind the implications of the new protocol for the whole Physics from a theoretical point of view, while from the purely experimental point of view we must consider the reality of the laboratory affected by innumerable noises due to all the gates involved for the purpose of implementing the different protocols. For this last reason, we need a thorough analysis in the presence of noise.

\subsection{Noiseless analysis}

The quantum teleportation begins with the distribution of the EPR pair $\left|\beta_{00}\right\rangle$ to Alice and Bob. This distribution constitutes the entanglement link between Alice and Bob, and after that, we continue with the complete sketch of quantum teleportation of Figure 4, where the green line indicates the border between the sides of Alice and Bob, that is, both extremes of the entanglement link. In Figure 4, a single fine line represents a wire carrying one qubit, while a double line represents a wire carrying one classical bit [24]. Besides, the classical channel is really a control classical channel for disambiguation purposes (as we will see below through two bits), while the entanglement link is really an entanglement data link. Besides, in this figure, the block with an $H$ represents a Hadamard's gate, and $\left|\beta_{00}\right\rangle \equiv\left|\Phi_{+}^{A \cup B}\right\rangle$ of Equation (10).

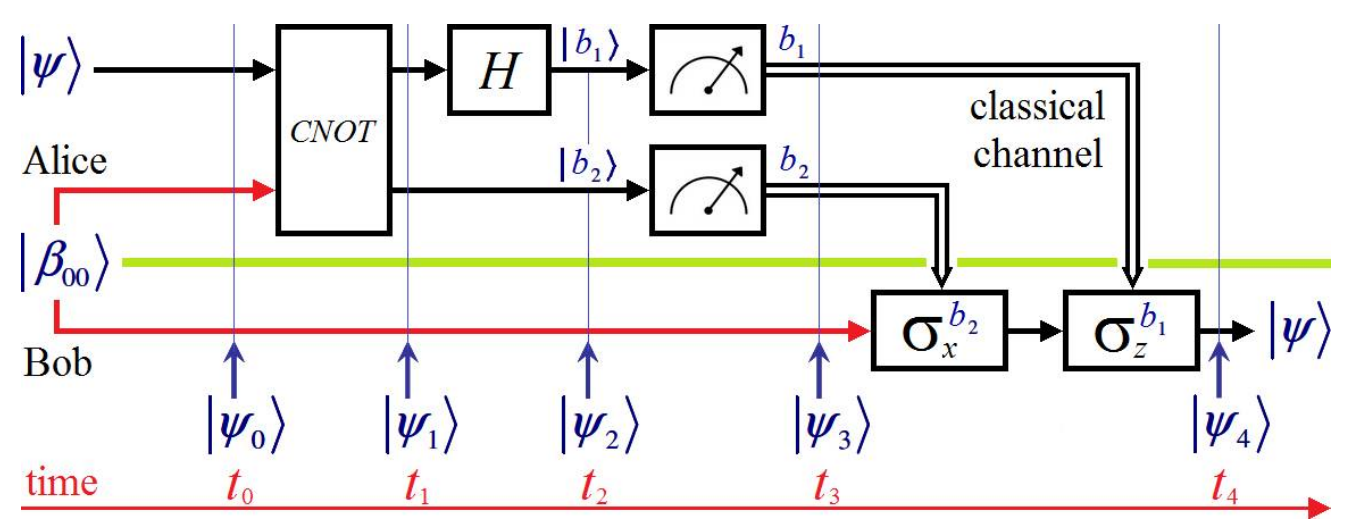

Fig.4 Standard Teleportation protocol using an EPR pair and two classical bits for disambiguation.

Now, If $|\psi\rangle=\alpha|0\rangle+\beta|1\rangle$ is an arbitrary state to be teleported with $|\alpha|^{2}+|\beta|^{2}=1$ and $\alpha \wedge \beta \in \mathbb{C}$ of a Hilbert space, then, the initial state (3-partite state) will be,

$$
\begin{aligned}
\left|\psi_{0}\right\rangle=|\psi\rangle \otimes\left|\beta_{00}\right\rangle=|\psi\rangle\left|\beta_{00}\right\rangle & =(\alpha|0\rangle+\beta|1\rangle) 1 / \sqrt{2}(|00\rangle+|11\rangle) \\
& =1 / \sqrt{2}[\alpha|0\rangle(|00\rangle+|11\rangle)+\beta|1\rangle(|00\rangle+|11\rangle)] \\
& =1 / \sqrt{2}[\alpha|000\rangle+\alpha|011\rangle+\beta|100\rangle+\beta|111\rangle]
\end{aligned}
$$

where for simplicity (and from here on) in a generic form $|x\rangle \otimes|y\rangle=|x\rangle|y\rangle$.

Now, CNOT gate is applied to Equation (15), 
$\left|\psi_{1}\right\rangle=\frac{1}{\sqrt{2}}[\alpha|000\rangle+\alpha|011\rangle+\beta|110\rangle+\beta|101\rangle]$

At this time, we apply a Hadamard's gate to the elements of Equation (16),

$$
\begin{aligned}
\left|\psi_{2}\right\rangle & =\frac{1}{2}\left[|00\rangle \sigma_{x}^{0} \sigma_{z}^{0}|\psi\rangle+|01\rangle \sigma_{x}^{1} \sigma_{z}^{0}|\psi\rangle+|10\rangle \sigma_{x}^{0} \sigma_{z}^{1}|\psi\rangle+|11\rangle \sigma_{x}^{1} \sigma_{z}^{1}|\psi\rangle\right] \\
& =\frac{1}{2}\left[\left|\Phi^{+}\right\rangle \sigma_{x}^{0} \sigma_{z}^{0}|\psi\rangle+\left|\Phi^{-}\right\rangle \sigma_{x}^{0} \sigma_{z}^{1}|\psi\rangle+\left|\Psi^{+}\right\rangle \sigma_{x}^{1} \sigma_{z}^{0}|\psi\rangle+\left|\Psi^{-}\right\rangle \sigma_{x}^{1} \sigma_{z}^{1}|\psi\rangle\right]
\end{aligned}
$$

Besides, Table I synthesizes the complete process of quantum teleportation, where Alice measures two of the possible qubits of the basis of Equation (10) and therefore she transmits the corresponding bits $b_{1}$ and $b_{2}$ via a classical channel to Bob. The quantum measurement process is imperative in order to make the wave-function of the original arbitrary state collapse since this is necessary to do not violate the No-Cloning Theorem. In other words, the quantum measurement process destroys the original arbitrary state [24].

TABLE I

ALICE'S SIDE: MEASUREMENT OF THE BASE, CLASSICAL TRANSMISSION OF BITS, AND THE COLLAPSE OF STATES, BOB'S SIDE: CLASSICAL RECEPTION OF BITS, GATES APPLICATION FOR THE FINAL RECOVERY OF THE ARBITRARY STATE.

\begin{tabular}{ccccc}
\hline \multicolumn{1}{c}{$\begin{array}{c}\text { Alice's } \\
\text { measurement }\end{array}$} & $\begin{array}{c}\text { Alice } \\
\text { transmits }\end{array}$ & $\begin{array}{c}\text { This happens with } \\
\text { probability }\end{array}$ & Collapsed state & Bob applies $\sigma_{x}^{b_{1}} \sigma_{z}^{b_{2}}$ \\
\hline$\left|\Phi^{+}\right\rangle \rightarrow 00$ & $\mathrm{~b}_{2} \mathrm{~b}_{1}=00$ & $\| \frac{1}{2} \sigma_{x}^{0} \sigma_{z}^{0}|\psi\rangle \|^{2}=\frac{1}{4}$ & $\left|\Phi^{+}\right\rangle \sigma_{x}^{0} \sigma_{z}^{0}|\psi\rangle$ & $\sigma_{x}^{0} \sigma_{z}^{0}|\psi\rangle=|\psi\rangle$ \\
$\left|\Psi^{+}\right\rangle \rightarrow 01$ & $\mathrm{~b}_{2} \mathrm{~b}_{1}=01$ & $\| \frac{1}{2} \sigma_{x}^{1} \sigma_{z}^{0}|\psi\rangle \|^{2}=\frac{1}{4}$ & $\left|\Psi^{+}\right\rangle \sigma_{x}^{1} \sigma_{z}^{0}|\psi\rangle$ & $\sigma_{x}^{1} \sigma_{z}^{0}|\psi\rangle=\sigma_{x}|\psi\rangle$ \\
$\left|\Phi^{-}\right\rangle \rightarrow 10$ & $\mathrm{~b}_{2} b_{1}=10$ & $\| \frac{1}{2} \sigma_{x}^{0} \sigma_{z}^{1}|\psi\rangle \|^{2}=\frac{1}{4}$ & $\left|\Phi^{-}\right\rangle \sigma_{x}^{0} \sigma_{z}^{1}|\psi\rangle$ & $\sigma_{x}^{0} \sigma_{z}^{1}|\psi\rangle=\sigma_{z}|\psi\rangle$ \\
$\left|\Psi^{-}\right\rangle \rightarrow 11$ & $\mathrm{~b}_{2} \mathrm{~b}_{1}=11$ & $\| \frac{1}{2} \sigma_{x}^{1} \sigma_{z}^{1}|\psi\rangle \|^{2}=\frac{1}{4}$ & $\left|\Psi^{-}\right\rangle \sigma_{x}^{1} \sigma_{z}^{1}|\psi\rangle$ & $\sigma_{x}^{1} \sigma_{z}^{1}|\psi\rangle=\sigma_{x} \sigma_{z}|\psi\rangle$ \\
\hline
\end{tabular}

At this point, it is important to mention that in literature there are several concerns regarding the implementation of teleportation protocols using a bigger or smaller dimensional commitment but always with two classical bits for disambiguation. An interesting example can be found in [28], which shows that the one-qubit teleportation can be considered as a state transfer between subspaces of the whole Hilbert space of an indivisible eight-dimensional system. However, this as well as the rest of the works that manipulate high dimensional quantum systems for the implementation of quantum teleportation protocols do it with two classical bits for disambiguation.

On Alice's side, the combination of the modules constituted by the following gates: CNOT, $H$ (Hadamard) and quantum measurement, constitute what is known as Bell State Measurement (BSM), while on Bob's side, its modules are unitary operations necessary for the reconstruction of the teleported state. What Alice did plus the transmission of the classic bits of disambiguation along with Bob's unitary operations is the clearest example of Local Operations and Classical Communication (LOCC) [29].

Finally, we must highlight as a fundamental contrast between this version of the quantum teleportation protocol with the following one, that here, i.e., in Equation (15) we make $|\psi\rangle \otimes\left|\beta_{00}\right\rangle$ and that the Kronecker product " $\otimes$ " is not commutative.

\subsection{Noisy analysis}

Starting again from Figure 4, and considering noise in the EPR pair by a disturbance of the shape

$$
\left|\beta_{00}\right\rangle_{n}=A|00\rangle+B|11\rangle
$$

where subscript $n$ means noise, and 
$|A|^{2}+|B|^{2}=1$, with $(A \neq B) \wedge(A \neq 1 / \sqrt{2}) \wedge(B \neq 1 / \sqrt{2})$

Then, repeating Equation (15) but with $\left|\beta_{00}\right\rangle_{n}$ instead of $\left|\beta_{00}\right\rangle$, we will have

$$
\begin{aligned}
\left|\psi_{0}\right\rangle=|\psi\rangle\left|\beta_{00}\right\rangle_{n} & =(\alpha|0\rangle+\beta|1\rangle)(A|00\rangle+B|11\rangle) \\
& =\alpha A|000\rangle+\beta A|100\rangle+\alpha B|011\rangle+\beta B|111\rangle
\end{aligned}
$$

Now, CNOT gate is applied to Equation (20),

$\left|\psi_{1}\right\rangle=\alpha A|000\rangle+\beta A|110\rangle+\alpha B|011\rangle+\beta B|101\rangle$

At this time, we apply a Hadamard's gate to the elements of Equation (21),

$$
\begin{aligned}
\left|\psi_{2}\right\rangle & =\frac{1}{\sqrt{2}}[\alpha A|000\rangle+\alpha A|100\rangle+\beta A|010\rangle-\beta A|110\rangle+\alpha B|011\rangle+\alpha B|111\rangle+\beta B|001\rangle-\beta B|101\rangle] \\
& =\frac{A}{\sqrt{2}}|00\rangle \alpha|0\rangle+\frac{B}{\sqrt{2}}|00\rangle \beta|1\rangle+\frac{A}{\sqrt{2}}|10\rangle \alpha|0\rangle-\frac{B}{\sqrt{2}}|10\rangle \beta|1\rangle+\frac{A}{\sqrt{2}}|01\rangle \beta|0\rangle+\frac{B}{\sqrt{2}}|01\rangle \alpha|1\rangle+\frac{B}{\sqrt{2}}|11\rangle \alpha|1\rangle-\frac{A}{\sqrt{2}}|11\rangle \beta|0\rangle \\
& =|00\rangle\left(\frac{A}{\sqrt{2}} \alpha|0\rangle+\frac{B}{\sqrt{2}} \beta|1\rangle\right)+|10\rangle\left(\frac{A}{\sqrt{2}} \alpha|0\rangle-\frac{B}{\sqrt{2}} \beta|1\rangle\right)+|01\rangle\left(\frac{B}{\sqrt{2}} \alpha|1\rangle+\frac{A}{\sqrt{2}} \beta|0\rangle\right)+|11\rangle\left(\frac{B}{\sqrt{2}} \alpha|1\rangle-\frac{A}{\sqrt{2}} \beta|0\rangle\right)
\end{aligned}
$$

From here, we follow a procedure similar to that of Table I but taking into account how sensitively the state will be affected by noise.

\section{Simplified Quantum Teleportation}

First, and unlike the previous one, the new protocol dispenses with a classic channel to transmit the disambiguation bits, as well as it does not require the use of the Pauli matrices of Bob's side in order to reconstruct the teleported state from the mentioned disambiguation bits. These simplifications are the reason for the title of this paper, that is, simplified protocol.

For the new protocol we will focus on Figure 5 using $\left|\beta_{00}\right\rangle \otimes|\psi\rangle$ instead of $|\psi\rangle \otimes\left|\beta_{00}\right\rangle$.

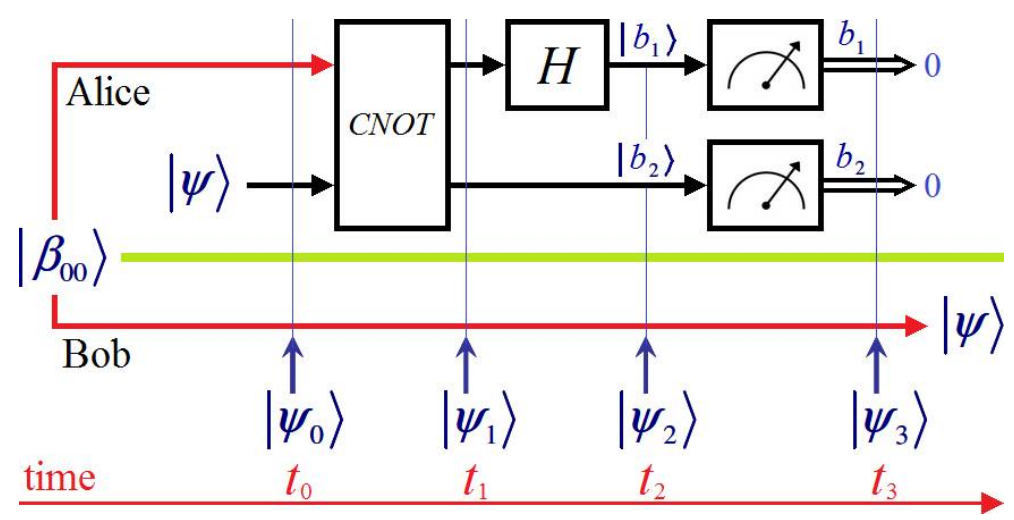

Fig.5 Simplified Teleportation protocol using an EPR pair but without classical bits for disambiguation.

\subsection{Noiseless analysis}

$$
\begin{aligned}
\left|\psi_{0}\right\rangle=\left|\beta_{00}\right\rangle|\psi\rangle & =\frac{1}{\sqrt{2}}(|00\rangle+|11\rangle)(\alpha|0\rangle+\beta|1\rangle) \\
& =\frac{1}{\sqrt{2}}[\alpha|000\rangle+\beta|001\rangle+\alpha|110\rangle+\beta|111\rangle]
\end{aligned}
$$

Now, CNOT gate is applied to Equation (23), 
$\left|\psi_{1}\right\rangle=\frac{1}{\sqrt{2}}[\alpha|000\rangle+\beta|001\rangle+\alpha|100\rangle+\beta|101\rangle]$

Applying a Hadamard's gate to the elements of Equation (24),

$$
\begin{aligned}
\left|\psi_{2}\right\rangle & =\frac{1}{2}[\alpha|000\rangle+\alpha|100\rangle+\beta|001\rangle+\beta|101\rangle+\alpha|000\rangle-\alpha|100\rangle+\beta|001\rangle-\beta|101\rangle] \\
& =\alpha|000\rangle+\beta|001\rangle \\
& =|00\rangle(\alpha|0\rangle+\beta|1\rangle)
\end{aligned}
$$

It is clear from Equation (25) that no disambiguation is necessary. Alice measures (where, both bits are always equal to zero independently of $|\psi\rangle$ ) in order to annul the entanglement and thus avoid violating the No-Cloning Theorem $[5,16]$. We can also see in Figure 5 that it is not necessary for Bob to apply any unitary transformation. This eliminates the classic channel that is responsible for making teleportation as a whole to be carried out in a time greater than zero, i.e., not being instantaneous.

\subsection{Noisy analysis}

For noisy EPR pairs we also resorted to Figure 5 and using the same version of Equations (18) and (19). Then, repeating Equation (23) but with $\left|\beta_{00}\right\rangle_{n}$ instead of $\left|\beta_{00}\right\rangle$, we will have

$$
\begin{aligned}
\left|\psi_{0}\right\rangle=\left|\beta_{00}\right\rangle_{n}|\psi\rangle & =(A|00\rangle+B|11\rangle)(\alpha|0\rangle+\beta|1\rangle) \\
& =A \alpha|000\rangle+B \alpha|110\rangle+A \beta|001\rangle+B \beta|111\rangle
\end{aligned}
$$

Now, we apply CNOT gate to Equation (26),

$$
\left|\psi_{1}\right\rangle=A \alpha|000\rangle+B \alpha|100\rangle+A \beta|001\rangle+B \beta|101\rangle
$$

Applying a Hadamard's gate to the elements of Equation (27),

$$
\begin{aligned}
\left|\psi_{2}\right\rangle & =\frac{1}{\sqrt{2}}[A \alpha|000\rangle+A \alpha|100\rangle+B \alpha|000\rangle-B \alpha|100\rangle+A \beta|001\rangle+A \beta|101\rangle+B \beta|001\rangle-B \beta|101\rangle] \\
& =\frac{A}{\sqrt{2}}|00\rangle(\alpha|0\rangle+\beta|1\rangle)+\frac{B}{\sqrt{2}}|00\rangle(\alpha|0\rangle+\beta|1\rangle)+\frac{A}{\sqrt{2}}|10\rangle(\alpha|0\rangle+\beta|1\rangle)-\frac{B}{\sqrt{2}}|10\rangle(\alpha|0\rangle+\beta|1\rangle) \\
& =\left[\left(\frac{A+B}{\sqrt{2}}\right)|00\rangle+\left(\frac{A-B}{\sqrt{2}}\right)|10\rangle\right](\alpha|0\rangle+\beta|1\rangle) \\
& =C(\alpha|0\rangle+\beta|1\rangle)
\end{aligned}
$$

where

$$
C=\left(\frac{A+B}{\sqrt{2}}\right)|00\rangle+\left(\frac{A-B}{\sqrt{2}}\right)|10\rangle
$$

The worst consequence of noise on the new protocol is that the teleported state loses its purity, that means, it would not be on Bloch's sphere, in the more general case, given that $C \neq 1$, but even so, the teleported state is recovered without problems and without disambiguation. This clearly indicates that the new protocol is much more robust (immune to noise) than the standard.

\section{Conclusions and Future Works}

In this work, we have presented a new quantum teleportation protocol in which we have eliminated the 
classical channel used currently for disambiguation, and since the classical channel is the weak link of standard teleportation in all matters relating to safety and speed, the novel seems to be an interesting alternative. We speak of security since quantum cryptography [30] [31] is much more robust than its classical counterpart, being its main tool the quantum key distribution (QKD) [5] [24] [30] [31]. On the other hand, in the standard protocol of quantum teleportation, the classical channel forces the communications system (as a complete unit) to have a speed of transmission equal to the speed of light, that is, the system is not instantaneous. In other words, in the case of an interplanetary communication, the traditional teleportation behaves (from the point of view of speed) as a current classic communications system. Instead, the new protocol is instantaneous since it does not require disambiguation through the use of a classic channel, gaining robustness (i.e., noise immunity) and security, and requiring fewer components for its implementation in a laboratory.

The experimental implementation of the new protocol will allow us to verify what is established in [23], which is an attempt to unify the two main pillars of Physics: the Theory of Relativity and Quantum Mechanics, as well as get a better understanding of the entanglement. This happens because the new protocol takes full advantage of the main attribute of entanglement: the instantaneity.

Furthermore, in the Quantum Communications work mentioned above [23] is demonstrated that a superluminal signaling [7] [8] is not necessary for entanglement to be instantaneous.

The future challenges in this area involve: a thorough study of what this work means in black hole theory, and, a formal and detailed analysis of the complete attributes of the entanglement and therefore of a quantum channel in terms of its bandwidth, channel capacity and information transit time for Quantum Communications [32]-[34]. Besides, the impact of the aforementioned verification on Quantum Internet [35] [36] and Super Dense Coding [5] [17] is evident.

Finally, and considering that there are already precedents of teleportation of energy [37] [38], and keeping in mind [23], an interesting question arises: can we teleport matter through exclusively quantum channels in a not too distant future?

\section{References}

1. Jaeger, G. 2009 Entanglement, Information, and the Interpretation of Quantum Mechanics. The Frontiers Collection. Springer-Verlag, Berlin. (doi:10.1007/978-3-540-92128-8_2)

2. Schrödinger, E. 1935 Die gegenwaertige Situation in der Quantenmechanik, Die Naturwissenschaften 23, 807. (doi:10.1007/978-3-663-14179-2_9)

3. Schrödinger, E. 1935 Discussion of probability relations between separated systems, Proc. Cambridge Philos. Soc. 32, 446. (doi:10.1017/S0305004100013554)

4. Einstein, A., Podolsky, B., Rosen, N. 1935 Can Quantum-Mechanical Description of Physical Reality Be Considered Complete? Physical Review. 47 (10): 777-780. (doi:10.1103/PhysRev.47.777)

5. Audretsch, J. 2007 Entangled Systems: New Directions in Quantum Physics. Wiley-VCH Verlag $\mathrm{GmbH} \& \mathrm{Co}$, Weinheim.

6. Einstein, A., Lorentz, H. A., Minkowski, H., Weyl, H. 1952 The Principle of Relativity: a collection of original memoirs on the special and general theory of relativity. Courier Dover Publications. NY.

7. Herbert, N. 1982 FLASH - A superluminal communicator based upon a new kind of quantum measurement. Foundations of Physics. 12(12), 1171-1179. (doi: 10.1007/BF00729622)

8. Eberhard, P. H. \& Ross, R. R. 1989 Quantum field theory cannot provide faster-than-light communication. Foundations of Physics Letters 2: 127. (doi:10.1007/BF00696109)

9. Bell, J. 1964 On the Einstein Podolsky Rosen paradox. Physics. 1:195. (doi:10.1103/PhysicsPhysiqueFizika.1.195)

10. Vaidman, L. 2014 Quantum theory and determinism. Quantum Stud.: Math. Found. Springer. (doi:10.1007/s40509-014-0008-4)

11. Dieks, D. 1982 Communication by EPR devices. Physics Letters A, vol.92, issue 6, 271-272. (doi:10.1016/0375-9601(82)90084-6)

12. Ghirardi, G. C., Grassi, R., Rimini, A., Weber, T. 1988 Experiments of the EPR Type Involving CP-Violation Do not Allow Faster-than-Light Communication between Distant Observers. Europhys. Lett. Vol.6, 95-100. (doi:10.1209/0295-5075/6/2/001) 
13. Aspect, A., Grangier, P., Roger, G. 1982 Experimental Realization of Einstein-Podolsky-RosenBohm Gedankenexperiment: A New Violation of Bell's Inequalities. Physical Review Letters. Vol. 49, Iss. 2, 91-94. (doi:10.1103/PhysRevLett.49.91)

14. Clauser, J. F., Horne, M. A., Shimony, A., Holt, R. A. 1969 Proposed experiment to test local hidden-variable theories. Physical Review Letters, Vol.23, No.15, 880-884. (doi:10.1103/PhysRevLett.23.880)

15. Hanson, R. 2005 Loophole-free Bell inequality violation using electron spins separated by 1.3 kilometers. Nature. 526: 682-686. (doi:10.1038/nature15759)

16. Wootters, W. K., Zurek, W. H. 1982 A single quantum cannot be cloned. Nature, 299, 802-803. (doi:10.1038/299802a0)

17. Bennett, C. H., Brassard, G., Grepeau, C., Jozsa, R. 1993 Teleporting an Unknown Quantum State via Dual Classical and Einstein-Podolsky-Rosen Channels. Phys. Rev. Lett. 70, 1895 (doi: 10.1103/PhysRevLett.70.1895)

18. Bouwmeester, D. et al. 1997 Experimental Quantum Teleportation. Nature, 390, 575-579. (doi:10.1038/37539)

19. Boschi, D. et al. 1998 Experimental Realization of Teleporting an Unknown Pure Quantum State via Dual Classical and Einstein-Podolsky-Rosen Channels. Phys. Rev. Lett., 80, 1121. (10.1103/PhysRevLett.80.1121)

20. Schlosshauer, M. 2005 Decoherence, the measurement problem, and interpretations of quantum mechanics. Reviews of Modern Physics. 76 (4): 1267-1305. (doi: 10.1103/RevModPhys.76.1267)

21. Busch, P., Lahti, P., Pellonpää, J. P., Ylinen, K. 2016 Quantum Measurement. Springer, NY.

22. Mastriani, M. 2018 Optimal Estimate of Quantum States. Journal of Applied Mathematics and Physics. Vol.6, No.6, 1363-1381. (doi:10.4236/jamp.2018.66114)

23. Mastriani, M. 2018 Quantum Communications, Relativistic Entanglement and the Theory of Dilated Locality. (https://hal.inria.fr/hal-01655231)

24. Nielsen, M. A., Chuang, I. L. 2004 Quantum Computation and Quantum Information. Cambridge University Press, Cambridge.

25. Bang, J., Ryu, J., Kaszlikowski, D. 2018 Fidelity deviation in quantum teleportation. (https://arxiv.org/abs/1801.06115)

26. Hofmann, H. F., Ide, T., Kobayashi, T., Furusawa, A. 2000 Fidelity and information in the quantum teleportation of continuous variables. (https://arxiv.org/abs/quant-ph/0003053)

27. Oh, S., Lee, S., Lee, H. W. 2002 Fidelity of Quantum Teleportation through Noisy Channels. Physical Review A, 66, 022316. (doi:10.1103/PhysRevA.66.022316)

28. Kiktenko, E. O., Fedorov, A. K., Manko, V. I. 2016 Teleportation in an indivisible quantum system. (https://arxiv.org/abs/1512.05168)

29. Kiktenko, E. O., Popov, A. A. and Fedorov, A. K. 2016 Bidirectional imperfect quantum teleportation with a single Bell state. (https://arxiv.org/abs/1602.01420)

30. Bennett, C.H., Brassard, G. 1984 Quantum cryptography: Public key distribution and coin tossing. Proc. IEEE Intern. Confer. Computers, Systems and Signal Processing, 175-179. (doi:10.1016/j.tcs.2014.05.025)

31. Anderson, R., Brady, R. 2013 Why quantum computing is hard - and quantum cryptography is not probably secure. (https://arxiv.org/abs/1301.7351)

32. Cariolaro, G. 2015 Quantum Communications. Springer International Publishing. NY.

33. Mishra, V. K. 2016 An Introduction to Quantum Communication. Momentum Press, NY.

34. Imre, S., Gyongyosi, L. 2012 Advanced Quantum Communications: An Engineering Approach. Wiley-IEEE Press, NY.

35. Kimble, H. J. 2018 The quantum internet. Nature, Vol.453, $1023-1030$. (doi:10.1038/nature07127)

36. Wehner, S. 2018 The quantum internet has arrived (and it hasn't): Networks that harness entanglement and teleportation could enable leaps in security, computing and science. Nature, 554, 289-292. (doi:10.1038/d41586-018-01835-3)

37. Hotta, M., Matsumoto, J., Yusa, G. 2010 Energy Entanglement Relation for Quantum Energy Teleportation. (https://arxiv.org/abs/1002.0200) 
38. Hotta, M. 2014 Quantum Energy Teleportation without Limit of Distance. (https://arxiv.org/abs/1305.3955) 